\title{
Geochemical evidence for provenance of Ordovician cherts in
} southeastern Australia

\author{
M. C. BRUCE \& I. G. PERCIVAL
}

Geological Survey of New South Wales, W.B. Clarke Geoscience Centre, 947-953 Londonderry Road, Londonderry, NSW 2753, Australia.

e-mail: michael.bruce@trade.nsw.gov.au

\section{SUPPLEMENTARY PAPER}

Australian Journal of Earth Sciences (2014) 61(7)

http://dx.doi.org/10.1080/08120099.2014.956792

Copies of Supplementary Papers may be obtained from the Geological Society of Australia's website (www.gsa.org.au), the Australian Journal of Earth Scienecs website (www.ajes.com.au) or from the National Library of Australia's Pandora archive (http://nla.gov.au/nla.arc-25194).

\section{SUPPLEMENTARY PAPER}

Appendix: Locality and biostratigraphic information for analysed chert samples. 


\section{SUPPLEMENTARY MATERIAL}

Appendix: Locality and biostratigraphic information for analysed chert samples.

\section{Oberon 1:100 000 sheet (Murray \& Stewart 2001)}

Murray \& Stewart locality R15975: GR 7563456258737

8830-SM Budhang; Paracordylodus gracilis conodont Zone.

\section{Goulburn 1:100 000 sheet (Percival \& Sherwin 2004)}

Sample 8828-AJJ-32.1 (Bungonia Road cutting): GR 7608856142425

Nattery Chert Member of Abercrombie Formation; Pygodus serra conodont Zone.

Sample 8828-AJJ-33.1 (Bungonia Road cutting): GR 7625566141950

Mummel Chert Member of Abercrombie Formation; Oepikodus evae conodont Zone.

Sample 8828-AJJ-171.1 (Mountain Ash road): GR 7592716142170

Nattery Chert Member of Abercrombie Formation; Pygodus serra conodont Zone.

Sample 8828-AJJ-191.1 (roadside subcrop): GR 7640866143240

Nattery Chert Member of Abercrombie Formation; Pygodus serra conodont Zone.

Sample 8828-DJP-239.1 (crest of spur): GR 7663116175003

Nattery Chert Member of Abercrombie Formation; (Pygodus serra conodont Zone).

Sample 8828-DJP-283.1 (near crest of knoll): GR 7658276171921

Nattery Chert Member of Abercrombie Formation; Pygodus serra conodont Zone.

Sample 8828-DJP-285.1 (beside fire trail): GR 7654796172382

Peach Tree Chert Member of Abercrombie Formation; Paroistodus horridus conodont Zone.

Sample 8828-DJP-287.1 (ridge top beside fire trail): GR 7648956170571

upper Abercrombie Formation; age: Darriwilian 3 to Gisbornian 1.

SAMPLE 8828-AJJ-64.1 (HUME HIGHWAY ROADCUT): GR 7614126151348

Mummel Chert Member of Abercrombie Formation; Oepikodus evae conodont Zone.

Sample 8828-ODT-201.1 (exposure in gully): GR 7402546151812

Nattery Chert Member of Abercrombie Formation; (Pygodus serra conodont Zone).

Sample 8828-ODT-203.1 (200m E of white shed): GR 7413326153647

Nattery Chert Member of Abercrombie Formation; (Pygodus serra conodont Zone).

Sample 8828-LS-120: GR 764623E 6164312N

Nattery Chert Member of Abercrombie Formation; Pygodus serra conodont Zone.

Sample 8828-GLLS-189: GR 761225E 6152072N

Nattery Chert Member of Abercrombie Formation; Pygodus serra conodont Zone.

Sample 8828-GLLS-193: GR 761488E 6153182N

Nattery Chert Member of Abercrombie Formation; (Pygodus serra conodont Zone). 
Bruce \& Percival 2014. Australian Journal of Earth Sciences 61(7) Supplementary Paper http://dx.doi.org/10.1080/08120099.2014.956792

\section{Sample 8828-LS-219: GR 7667556174060}

Nattery Chert Member of Abercrombie Formation; Pygodus serra conodont Zone.

\section{Gunning 1:100 000 sheet (Percival \& Sherwin 2003)}

Sample 8728-AYW-20 (roadcut, S of Rugby-Bevendale Road): GR 6877906176362

Peach Tree Chert Member of Abercrombie Formation; Paroistodus horridus conodont Zone.

Sample 8728-AYW-155 (1.25 km N of "Coolong" homestead): GR 6963986161232 Mummel Chert Member of Abercrombie Formation; Oepikodus evae conodont Zone.

Sample 8728-ODT-481 ( 100 m N of Yass River) GR 6911466135781

Nattery Chert Member of Abercrombie Formation; Pygodus serra conodont Zone.

\section{Taralga 1:100 000 sheet (Percival \& Sherwin 2005)}

Sample 8829-ODT-37.1 (Oaky Creek) GR 7474336217083

Peach Tree Chert Member of Abercrombie Formation; Paroistodus horridus conodont Zone.

Sample 8829-ODT-38.1 (Silent Creek fire trail): GR 7471536216792

Peach Tree Chert Member of Abercrombie Formation; Paroistodus horridus conodont Zone.

Sample 8829-ODT-82.1: GR 7469896214443

Nattery Chert Member of Abercrombie Formation; Pygodus serra conodont Zone.

Sample 8829-ODT-92.1: GR 7535476215098

Peach Tree Chert Member of Abercrombie Formation; Paroistodus horridus conodont Zone.

Sample 8829-ODT-543.1: GR 7534616221284

Peach Tree Chert Member of Abercrombie Formation; Paroistodus horridus conodont Zone.

Sample 8829-ODT-802.1: GR 7544906216946

Mummel Chert Member of Abercrombie Formation; Oepikodus evae conodont Zone. Sample 8829-

LJS-179.1: GR 7685066179610

Peach Tree Chert Member of Abercrombie Formation; Paroistodus horridus - Spinodus spinatus conodont Zone.

Sample 8829-MMS-104.1 (ridge above Woodduck Creek): GR 7486756192700

Mummel Chert Member of Abercrombie Formation; Oepikodus evae conodont Zone.

\section{Braidwood 1:100 000 sheet (Percival \& Sherwin 2008)}

Sample 8827-KEB-102.3: GR 7670376120096

?Mummel Chert Member of Abercrombie Formation; Paracordylodus gracilis conodont Zone.

Sample 8827-KEB-390.1 (hill, Nadgigomar NR): GR 7572456103976

Mummel Chert Member of Abercrombie Formation; Oepikodus evae conodont Zone. 
Sample 8827-ODT-924.1: GR 7666726087481

Mummel Chert Member of Abercrombie Formation; Oepikodus evae conodont Zone.

Sample 8827-ODT-1019.1: GR 7722486088281

Peach Tree Chert Member of Abercrombie Formation; Paroistodus horridus - Spinodus spinatus conodont Zone.

Sample 8827-ODT-1022.1: GR 7710886088744

Mummel Chert Member of Abercrombie Formation; Oepikodus evae conodont Zone.

Sample 8827-ODT-1137.1: GR 7617316088771

lower Abercrombie Formation; Early Ordovician age.

Sample 8827-JAF-107.1: GR 7669116121750

?Mummel Chert Member of Abercrombie Formation; Paracordylodus gracilis conodont Zone.

Sample 8827-JAF-109.1: GR 7673186122928

Mummel Chert Member of Abercrombie Formation; Oepikodus evae conodont Zone.

Sample 8827-JAF-118.1: GR 7688786123190

Peach Tree Chert Member of Abercrombie Formation; Paroistodus horridus conodont Zone.

Sample 8827-JAF-224.1: GR 7663046113097

Peach Tree Chert Member of Abercrombie Formation; Paroistodus horridus conodont Zone.

\section{Sussex 1:100 000 sheet (Percival 2006b)}

Sample 8135-GRB-461.1: GR 4282476528512

Narrawa Formation; Oepikodus evae conodont Zone.

Sample 8135-GRB-497.1: GR 4503276525457

Ballast Formation; Pygodus serra conodont Zone.

Sample 8135-SJT-270.1: GR 4188286565921

Narrawa Formation; Oepikodus evae conodont Zone.

Sample 8135-SJT-401.1: GR 4094246521253

Narrawa Formation; Oepikodus evae conodont Zone.

Sample 8135-SJT-423.1: GR 4123036534952

Ballast Formation; Pygodus serra conodont Zone.

Sample 8135-SJT-427.1: GR 4189796539570

Ballast Formation; Pygodus serra conodont Zone.

\section{Byrock 1:100 000 sheet (Percival 2007)}

Sample 8136-SJT-25.2: GR 4075026582151

Narrawa Formation; Oepikodus evae conodont Zone. 


\section{Tantangara 1:100 000 sheet (Cam Quinn, unpublished data)}

\section{Sample 8626-CDQ-HH2: GR 6442506040670}

Stratigraphic unit uncertain, but probably correlative of the Nattery Chert Member, Abercrombie Formation. Presence of Pygodus sp. and Spinodus spinatus suggests most likely age is Darriwilian 3-4.

\section{Tumut 1:100 000 sheet (Cam Quinn, unpublished data)}

Sample 8527-CDQ-255: (Ridge immediately W of Tumut River) GR 6088406115770

Stratigraphic unit uncertain, but probably correlative of the upper Abercrombie Formation. Presence of Pygodus sp. suggests age range from late Darriwilian to earliest Gisbornian.

Sample 8257-CDQ-382: GR 6052716115392

Stratigraphic unit uncertain, but probably correlative of the uppermost Abercrombie Formation.

Pygodus anserinus conodont Zone (Darriwilian 4 to Gisbornian 1).

\section{Port Macquarie Block (Och, Percival \& Leitch 2007)}

Sample 9435-Ptmk-432: strongly recrystallised grey-black chert outcrop at Tacking Point. Age: most likely Late Ordovician (Gisbornian - Eastonian).

Sample 9435-Ptmk-436: Orange-brown chert from Flynns Beach.

Age: most likely ranging from late Darriwilian to early Gisbornian.

\section{Narooma Chert (Glen, Stewart \& Percival 2004)}

Grid references (AGD66) (estimates only) for sites located on the $2^{\text {nd }}$ edition (1989) Narooma 1:25 000 topographic sheet (8925-4-S) and on the $2^{\text {nd }}$ edition (1988) Central Tilba 1:25 000 topographic sheet (8925-3-N).

Sample 8925-N19: GR 238060mE 5984560mN (Wagonga Scenic Drive).

Paracordylodus gracilis conodont Zone (late Lancefieldian to late Bendigonian).

Sample 8925-N35: GR 241400mE 5980500mN (highway south of Corunna Bridge).

Paracordylodus gracilis conodont Zone (late Lancefieldian to late Bendigonian).

Sample 8925-N42: GR 241770mE 5988890mN (quarry off Centenary Drive).

Pygodus serra conodont Zone (late Darriwilian, Da3-4).

Sample 8925-N82B: GR 237300mE 5992825mN (Chert Hill, Nobles).

Oepikodus evae conodont Zone (Bendigonian, most likely Be2-3).

Sample 8925-N96-2: GR 240325mE 5991880mN (outcrops on Princes Highway).

Age: Furongian (late Cambrian).

Sample 8925-N96-3: GR 240350mE 5991890mN.

?Paracordylodus gracilis conodont Zone (late Lancefieldian to early Bendigonian). 
Sample 8925-N108: GR 237560mE 5987948mN (on north shore of Wagonga Inlet).

Spinodus spinatus conodont Zone (mid Darriwilian, Da2-3).

Sample 8925-N110: GR 236853mE 5988858mN.

Paracordylodus gracilis conodont Zone (late Lancefieldian to late Bendigonian).

Sample 8925-PL2924B: GR 240640mE 5991000mN (on Highway just north of Narooma).

Paracordylodus gracilis conodont Zone (late Lancefieldian to late Bendigonian).

Sample 8925-Kianga 4: GR 241500mE 5989750mN (road near Kianga Point).

Pygodus anserinus conodont Zone, latest Darriwilian (late Da4) to early Gisbornian (Gi1).

\section{REFERENCES (ADDITIONAL TO THOSE CITED IN MAIN TEXT)}

Percival I. G. \& SheRWIN L. 2003. Palaeontological Determinations, Gunning 1:100 000 sheet. Geological Survey of New South Wales, Report GS 2003/524. 9pp.

Percival I. G. \& Sherwin L. 2004. Palaeontological Determinations, Goulburn 1:100 000 sheet. Geological Survey of New South Wales, Report GS 2004/457. 33 pp.

Percival I. G. \& SheRWIN L. 2005. Palaeontological Determinations, Taralga 1:100 000 sheet. Geological Survey of New South Wales, Report GS 2005/750. 25 pp.

Percival I. G. \& Sherwin L. 2008. Palaeontological Determinations, Braidwood 1:100 000 